\title{
The trajectory of a photon emitted by an accelerating light source. The apparent motion of a light source
}

John Deligiannis ( $\square$ john.del.astro@gmail.com )

National and Kapodistrian University of Athens https://orcid.org/0000-0001-9536-3311

Article

Keywords: Special Relativity, General Relativity, Gravity

Posted Date: September 7th, 2021

DOl: https://doi.org/10.21203/rs.3.rs-851282/v1

License: (c) (1) This work is licensed under a Creative Commons Attribution 4.0 International License.

Read Full License 


\title{
The trajectory of a photon emitted by an accelerating light source. The apparent motion of a light source
}

\author{
John Deligiannis \\ Department of Physics, National and Kapodistrian University of Athens
}

August 26, 2021

\begin{abstract}
In this article the concept of "tachy-photons" is introduced. The tachy-photons are photons emitted by an accelerating light source. The tachy-photons can travel faster than the speed of light, but their average speed is equal to the speed of light. Using the trajectories of tachy-photons, the apparent motion of an accelerating light source is calculated. This apparent motion of the light source is dramatically different from its actual motion.
\end{abstract}

Keywords: Special Relativity, General Relativity, Gravity

\section{Introduction}

Consider a sound source on the floor of a moving train travelling at a constant speed (Fig. 1 (I)). At some point, the sound source emits a sound pulse vertically upwards. The trajectory of the sound pulse, as seen by an observer inside the train at the point $A$, is the straight line in Fig. 1 (I). Therefore, at some point, the sound pulse will reach the point $B$. This occurs because the sound pulse is "pushed" by the propagation medium (the air) inside the train. So we could say that the sound pulse "follows" the sound source.

Now consider a light source on the floor of a moving train travelling at a constant speed (Fig. 1 (II)). At some point, the light source emits a light pulse vertically upwards. It is known from the theory of Special Relativity, that the trajectory of the light pulse, as seen by an observer 

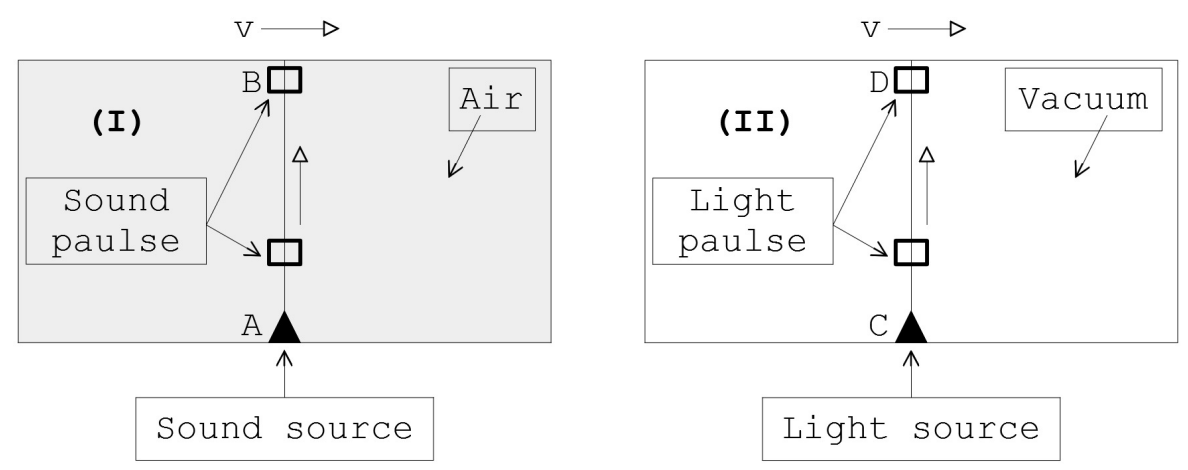

Figure 1: (I) The sound source (the triangular mark) on the floor of the train, emits a sound pulse (the square mark) vertically upwards. There is air in the space inside the train. (II) The light source (the triangular mark) on the floor of the train emits a light pulse (the square mark) vertically upwards. The space inside the train is vacant.

inside the train at the point $C$, is the straight line in Fig. 1 (II). Therefore, at some point the light pulse will reach the point $D$. This occurs because the train is an inertial reference frame. If we think about it, something impressive and strange is happening. The light pulse "follows" the light source even though it is not "pushed" by any propagation medium (the light propagates in the vacuum). The light pulse has no obvious reason to follow its source. It is logical to conclude that the above property doesn't depend on the nature of the reference frame. It is a property that arises from the fact that the light propagates in the vacuum. Therefore, we can assume that the above property also occurs in a non-inertial reference frame.

\section{Lorentz-type transformations of non-inertial frames}

Suppose that the light source in Fig. 2 is accelerating in a straight line and at $t=0$ emits a photon perpendicular (in its reference frame) to its displacement. If we accept that an emitted

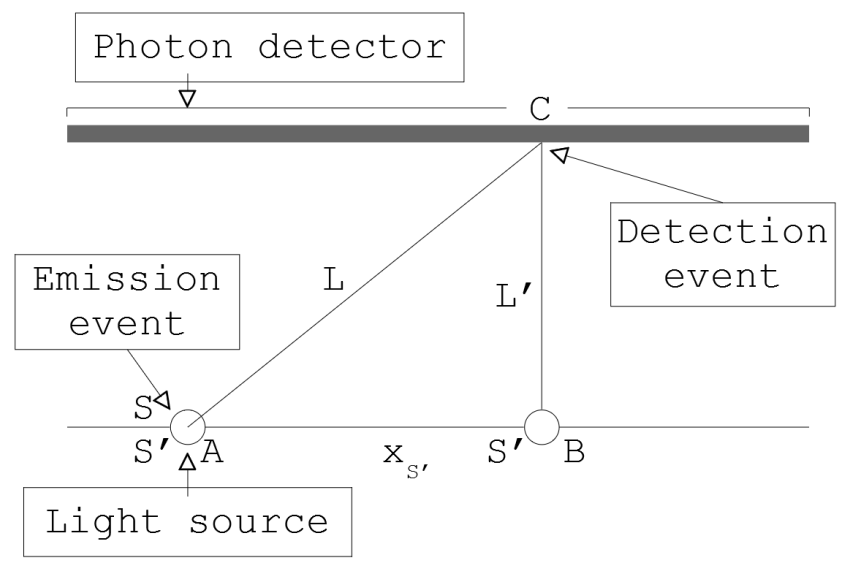

Figure 2: Time delay triangle. $S$ the fixed reference frame, $S^{\prime}$ the reference frame of light source.

photon "follows" the light source, as described in the previous section, then it will reach the 
point $C$ in time $\Delta t^{\prime}=L^{\prime} / c(c$, the speed of light). This is the time period between the emission event and the detection event for an accelerating observer at the point $S^{\prime}$. For the observer at the point $A$ the time period between the emission event and the detection event is $\Delta t=L / c$. Working analogously to the Einstein's triangular time-delay diagram, ${ }^{[1]}$ we have that:

$$
\begin{gathered}
L^{2}=L^{\prime 2}+x_{S^{\prime}}{ }^{2} \Rightarrow \\
c^{2} \Delta t^{2}=c^{2} \Delta t^{\prime 2}+x_{S^{\prime}}{ }^{2} \Rightarrow \\
c^{2} \Delta t^{2}=c^{2} \Delta t^{\prime 2}+\left(\frac{x_{S^{\prime}}}{\Delta t}\right)^{2} \Delta t^{2} \Rightarrow \\
\Delta t^{\prime}=\frac{1}{\gamma(\tilde{v})} \Delta t, \gamma(\tilde{v})=1 / \sqrt{1-(\tilde{v} / c)^{2}}, \tilde{v}=x_{S^{\prime}} / \Delta t
\end{gathered}
$$

If we assume that initially the clocks of the two observers are synchronized to zero, then the time delay equation Eq. 1 becomes:

$$
t_{S^{\prime}}^{\prime}=\frac{1}{\gamma(\tilde{v})} t_{S} \quad, \quad \gamma(\tilde{v})=1 / \sqrt{1-(\tilde{v} / c)^{2}} \quad, \quad \tilde{v}=x_{S^{\prime}} / t_{S}
$$

Eq. 2 has a similar form to the expression for the time dilation of Special Relativity. Thus, we can conclude that the coordinate transformations that connect the time and space coordinates of an accelerating reference frame with the coordinates of a fixed one, must have the following Lorentz-type form:

$$
\begin{gathered}
x^{\prime}=\gamma(\tilde{v})(x-\tilde{v} t) \\
y^{\prime}=y, z^{\prime}=z \\
t^{\prime}=\gamma(\tilde{v})\left(t-\frac{\tilde{v} x}{c^{2}}\right) \\
\tilde{v}=\frac{x_{S}^{\prime}}{t}
\end{gathered}
$$

Let's see an interesting symmetry compared to the Galilean transformation of Classical Physics. Let's consider a classical reference frame $S^{\prime}$ moving on the $x$ axis with non-constant speed. Using simple Geometry, it is obvious that:

$$
\begin{gathered}
x^{\prime}=x-x_{S^{\prime}}, y^{\prime}=y, t^{\prime}=t \Rightarrow \\
x^{\prime}=x-\frac{x_{S^{\prime}}}{t} t, y^{\prime}=y, t^{\prime}=t \Rightarrow \\
x^{\prime}=x-\tilde{v} t, y^{\prime}=y, t^{\prime}=t
\end{gathered}
$$


Eq. 7 has a similar form to the Galilean transformation $x^{\prime}=x-v t, y^{\prime}=y, t^{\prime}=t$.

\section{The equivalence principle and the light source's motion}

It is known from the equivalence principle that an accelerating frame with constant acceleration $\vec{a}$ is equivalent to a gravitational field with a gravitational acceleration equal to $\vec{g}=-\vec{a}$. But, which situation exactly does the equivalence principle describe? Consider two observers,

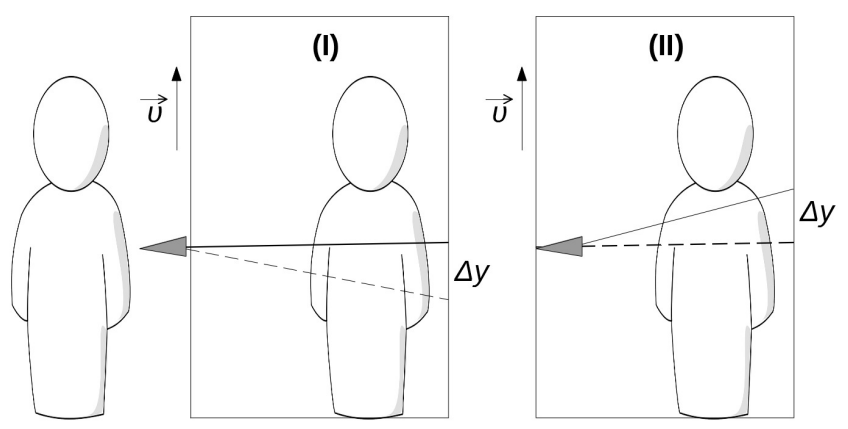

Figure 3: An elevator moves upwards at a constant speed $\vec{v}$. (I) The photon's source is fixed outside the elevator. (II) The photon's source is nailed to the left side of the elevator. The solid lines are the trajectories of the photon as seen by an observer outside the elevator. The dashed lines are the trajectories of the photon as seen by an observer inside the elevator.

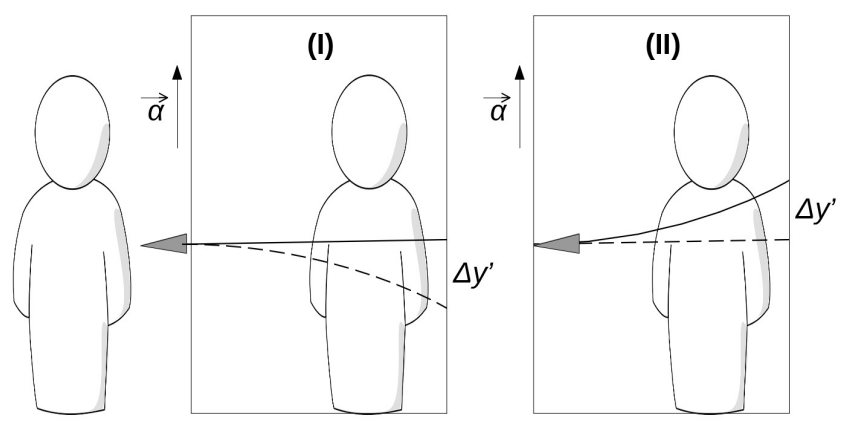

Figure 4: An elevator moves upwards with constant acceleration $\vec{a}$. (I) The photon's source is fixed outside the elevator. (II) The photon's source is nailed to the left side of the elevator. The solid lines are the trajectories of the photon as seen by an observer outside the elevator. The dashed lines are the trajectories of the photon as seen by an observer inside the elevator.

the one inside an elevator moving upwards at a constant speed $\vec{v}$ and the other outside the elevator, as shown in Fig. 3. Suppose that outside the elevator there is a fixed photons' source (Fig. 3 (I)). For the observer outside the elevator, the photon's trajectory is a horizontal line (the solid line in Fig. $3(\mathrm{I})$ ). For the observer inside the elevator, the photon's trajectory is a straight line sloping downwards (the dashed line in Fig. 3 (I)). Suppose now that the light source is nailed to the elevator's left side (Fig. 3 (II)). In this case, for the observer outside the elevator, the photon's trajectory is a straight line sloping upwards (the solid line in Fig. 
3 (II)). For the observer inside the elevator, the photon's trajectory is a horizontal line (the dashed line in Fig. 3 (II)). The theory of Special Relativity predicts the trajectories of Fig. 3. Therefore, it is known from the framework of Special Relativity that the light source's motion (as described above) affects the trajectory of the emitted photon.

Suppose that the elevator is moving upwards with a constant acceleration $\vec{a}$ and we execute the previous experiments (Fig. 4). Then, it is reasonable to conclude that the trajectories will be pretty much the same compared to the trajectories in Fig. 3, with the difference that the non-horizontal lines will be curved. So, the equivalence principle describes only the curved trajectory in Fig. 4 (I). The equivalence principle doesn't describe the curved trajectory in Fig. 4 (II). This trajectory can be described by using the Lorentz-type transformations of non-inertial reference frames.

\section{The trajectory of a photon emitted by an accelerating light source}

Suppose that the light source in Fig. 2 accelerates with a constant acceleration $a$ and initially has no speed $\left(v_{0}=0\right)$. So, the light source's equation of motion is $x_{S^{\prime}}=a t^{2} / 2$. At time $t=0$ a photon is emitted from the light source perpendicular (in the light source's reference frame) to its displacement. According to the consideration of Section 2, the photon "follows" the light source. This means that the coordinate $x^{\prime}$ of the emitted photon is equal to zero $\left(x^{\prime}=0\right)$. From Eq. 3 we have that the coordinate $x$ of the emitted photon is

$$
x=\frac{1}{2} a t^{2}
$$

The coordinate $y^{\prime}$ of the emitted photon is

$$
y^{\prime}=c t^{\prime}
$$

From Equations 4, 5, 8 and 9 we have that the coordinate $y$ of the emitted photon is

$$
y=c t \sqrt{1-\frac{a^{2} t^{2}}{4 c^{2}}}
$$




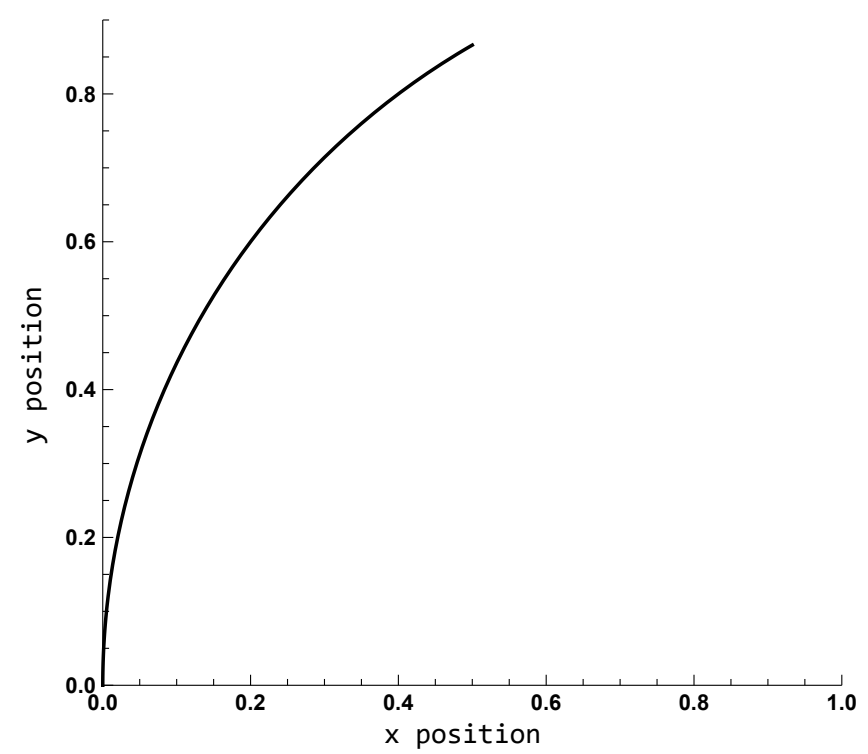

Figure 5: The trajectory of a photon emitted by an accelerating light source. $(0 \leq t \leq c / a, a=1, c=1)$

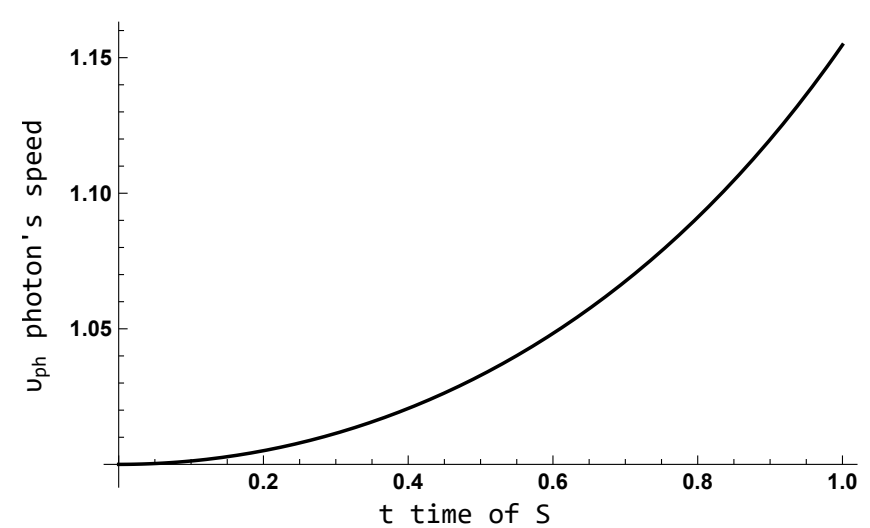

Figure 6: The speed of emitted photon versus the time $\mathrm{t}$ of reference frame $S .(0 \leq t \leq c / a, a=1, c=1)$

Solving Eq. 8 in terms of $t$ and substituting it into Eq. 10, we have that the trajectory of the emitted photon, as seen by an observer at the point $S$, is a circle with radius $c^{2} / a$.

$$
\left(x-\frac{c^{2}}{a}\right)^{2}+y^{2}=\left(\frac{c^{2}}{a}\right)^{2}
$$

From Equations 8 and 10, we have that the speed of the emitted photon is

$$
v_{p h}=\frac{c}{\sqrt{1-\frac{a^{2} t^{2}}{4 c^{2}}}}=\gamma(\tilde{v}) c
$$

The photon reaches its maximum speed at time $t_{0}=c / a$, when the light source reaches the speed of light.

$$
v_{p h(\max )}=\frac{2 c}{\sqrt{3}} \approx 1.1547 c
$$


From Eq. 8 and Eq. 10 we can calculate the average speed of light from time $t=0$ to time $t$.

$$
\begin{gathered}
<\vec{u}>=(x / t) \hat{x}+(y / t) \hat{y} \Rightarrow \\
<\vec{u}>=(a t / 2) \hat{x}+c \sqrt{1-\left(a^{2} t^{2}\right) /\left(4 c^{2}\right)} \hat{y} \Rightarrow \\
|<\vec{u}>|=c
\end{gathered}
$$

So, the photon's average speed is equal to c. Figures Fig. 5 and Fig. 6 show respectively the trajectory and the speed of the emitted photon from time $t=0$ to time $t=t_{0}=c / a$ when light source reaches the speed of light. It is well known that the light, as an electromagnetic wave, is described using the Classical Wave Equation. The wave equation in one dimension is $\partial^{2} \psi / \partial x^{2}-\left(1 / c^{2}\right) \partial^{2} \psi / \partial t^{2}=0$, where $c$ is the constant wave speed, $\psi=f(x-c t)+f(x+c t)$ is the wave form and $c=\lambda f$ is the law of wave propagation. The wave equation can also describe waves that travel at a constant average speed $\langle c\rangle$. In this case the wave equation is $\partial^{2} \psi / \partial x^{2}-\left(1 /<c>^{2}\right) \partial^{2} \psi / \partial t^{2}=0$, the wave form is $\psi=f(x-<c>t)+f(x+<c>t)$ and the law of wave propagation is $\langle c\rangle=\lambda f$.

\section{The Einstein's elevator as an evidence of the photon's circular trajectory}

A well-known accelerating reference frame is the frame of a free-falling elevator in a weak homogeneous gravitational field (Einstein's elevator ${ }^{[2]}$ ). On the one side of the elevator, there is a light source that emits photons to the other side. If the length of the elevator is $L$ (Fig. 7) then the time it takes a photon to reach the other side of the elevator is approximately $t_{1} \approx L / c$. The time $t_{1}$ is a tiny amount of time, so we can conclude that the curvature of the photon's trajectory is tiny too. So, we can accept that the photon's trajectory is approximately a tiny part ( $A B$ trajectory of Fig. 7$)$ of a huge circle. From Fig. 7, using the Pythagorean theorem we have that

$$
\begin{gathered}
R^{2}=L^{2}+(R-H)^{2} \Rightarrow \\
R=\frac{H}{2}+\frac{L^{2}}{2 H} \Rightarrow \\
R \approx \frac{g t_{1}^{2} / 2}{2}+\frac{\left(c t_{1}\right)^{2}}{2 g t_{1}^{2} / 2} \Rightarrow
\end{gathered}
$$




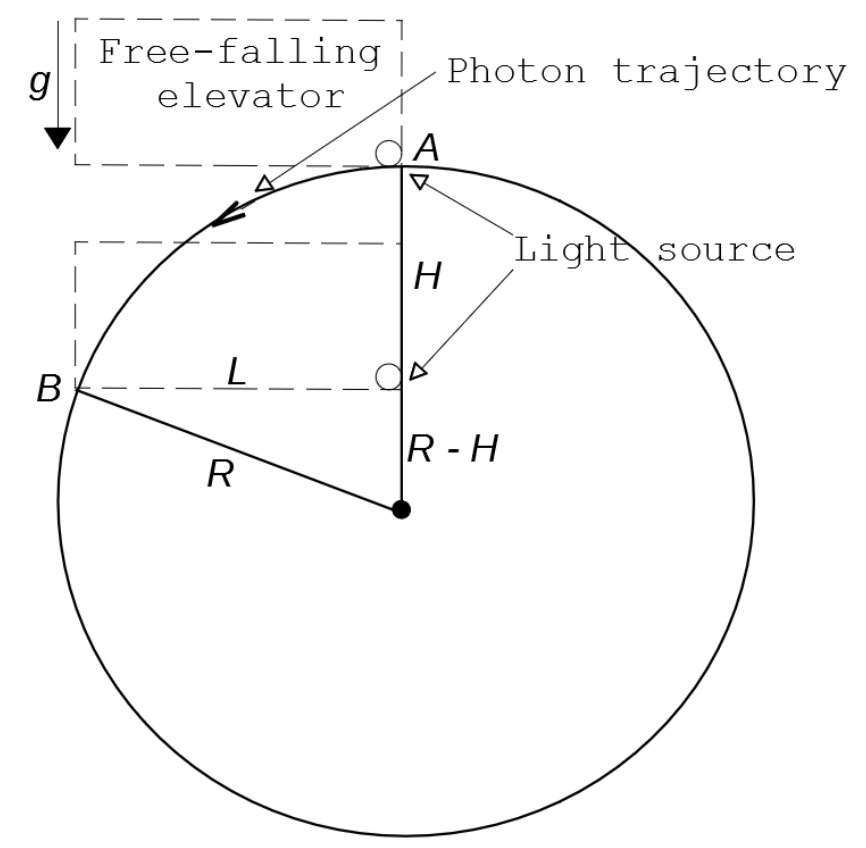

Figure 7: Free-falling elevator in a weak homogeneous gravity field.

$$
R \approx \frac{1}{4} g t_{1}^{2}+\frac{c^{2}}{g}
$$

Finally, under the consideration that $t_{1}$ is a tiny amount of time, we can conclude that the radius $R$ of the circle is approximately

$$
R \approx \frac{c^{2}}{g}
$$

The above radius is equal to the radius of the photon's trajectory predicted in Section 4 . This methodology is not a proof, but an evidence that the photon's trajectory predicted in Section 4 is correct.

\section{The apparent motion of the light source}

Consider a light source moving on the x-axis with constant acceleration $a$ and every time it increases its speed by $0.1 c$, it emits a photon perpendicular (in the light source's reference frame) to its displacement. Working analogously to Section 4 we can plot the photon's trajectory for every emission (Fig. 8). From Fig. 8 we can see that the trajectories of the emitted photons are constantly moving away from each other. Let's study two photons (the circles marks in Fig. 8) emitted when the light source had speed $u_{0}=0.2 c$ and $u_{0}=0.3 c$ respectively. The displacement of the light source is $\Delta x=x_{B}-x_{A}=0.046-0.022=0.024$. The apparent 


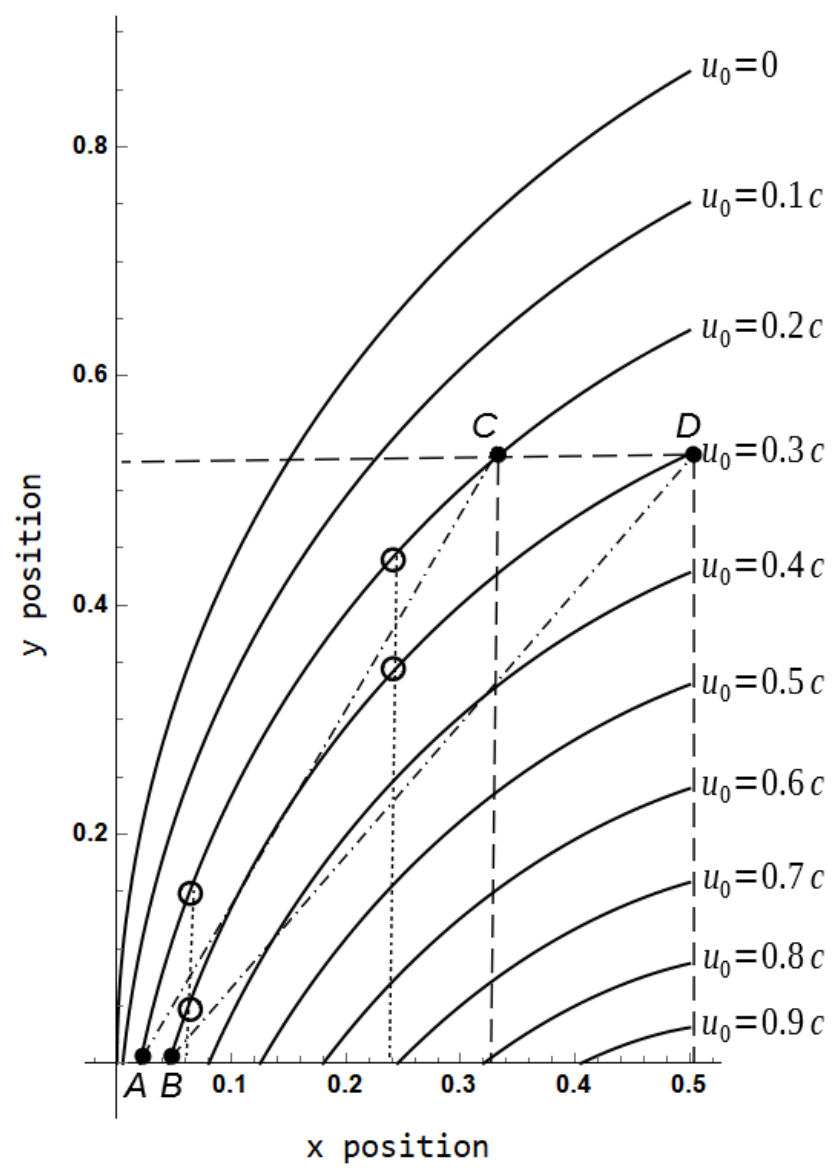

Figure 8: The light source is moving on the $\mathrm{x}$-axis with constant acceleration $a$ and every time it increases its speed by $0.1 c$, it emits a photon perpendicular (in the light source's reference frame) to its displacement. The trajectory of each photon is a time-parametric plot of equations $x-x_{0}=\tilde{v} t$ and $y=c t / \gamma(\tilde{v})$, which are derived by substituting $x^{\prime}=0$ into Eqs. 3, 4, 5 and 9 , where $\tilde{v}=u_{0}+a t / 2$ as result of Eq. $6, x_{0}=u_{0}^{2} /(2 a)$ and $0<t<\left(c-u_{0}\right) / a$. $(a=1, c=1)$

displacement is calculated when the two photons reach the points $C$ and $D$ respectively. So, the apparent displacement is $\Delta x_{\text {apparent }}=x_{D}-x_{C}=0.502-0.325=0.177\left(\Delta x_{\text {apparent }} / \Delta x \approx 7.4\right)$.

The average speed of the light source for the displacement $\Delta x=x_{B}-x_{A}$ is $\langle u\rangle=0.25 c$. The emitted photon from the point $A$ is travelling at constant average speed $c$. Thus, the time required for the photon to reach the point $C\left(y_{C}=0.53\right)$ is

$$
\Delta t_{1}=\frac{A C}{c}=\frac{1}{c} \sqrt{\left(x_{C}-x_{A}\right)^{2}+y_{C}^{2}}
$$

The emitted photon from the point $B$ is travelling at constant average speed $c$. Thus, the time required for the photon to reach the point $D\left(y_{D}=0.53\right)$ is

$$
\Delta t_{2}=\frac{B D}{c}=\frac{1}{c} \sqrt{\left(x_{D}-x_{B}\right)^{2}+y_{D}^{2}}
$$


The time required for the light source to travel the distance $\Delta x=x_{B}-x_{A}$ is

$$
\Delta t_{0}=\frac{x_{B}-x_{A}}{<u>}
$$

Therefore, the average apparent speed of the light source is calculated when the two photons reach the points $C$ and $D$ respectively.

$$
<u>_{\text {apparent }}=\frac{x_{D}-x_{C}}{\Delta t_{2}-\Delta t_{1}+\Delta t_{0}} \approx 0.96 c
$$

\section{Conclusions}

An emitted photon by an accelerating (with constant acceleration) light source can reach a speed $15.47 \%$ higher than the speed of light. Thus, such a photon can be called a "tachyphoton". The average speed of tachy-photons is equal to $c$. As it is shown in the end of Section 4, these results don't violate the Maxwell's equations of Electromagnetism. The trajectory of tachy-photons is a part of a circle with a radius equal to $c^{2} / a$, where $c$ is the speed of light and $a$ is the light source's constant acceleration. The idea that a photon under a mechanism can travel faster than the speed of light is strange. If we think about it, the idea that a photon travels at the speed of light is strange too. In the context of Special Relativity, an observer travelling at the speed of light will see a universe of infinite mass and zero length. Thus, the speed of light itself appears strange properties in the context of Special Relativity.

The result of Section 6 is that tachy-photons emitted by an accelerating light source give an apparent motion for the source. In this apparent motion the measurements of the displacement and speed of the light source are dramatically different from the actual values. It is known that the universe is expanding at an accelerating rate. This phenomenon has forced the astronomers to introduce the concepts of Dark Matter and Dark Energy. ${ }^{[3]}$ The composition of these two concepts is an unsolved problem in Physics. But in the context of tachy-photons, the measurements of the speed and distance of the stars are completely misleading. Maybe, according to this logic, the concepts of Dark Matter and Dark Energy are not needed or their amounts are much smaller than those calculated by cosmological models. Maybe the cosmos is an illusion. Of course this hypothesis needs more study and research. 


\section{References}

[1] S. Adams, An introduction to space-time physics, 2nd edn. (Taylor \& Francis, Philadelphia, 1997)

[2] J. R. Brown, The Laboratory of the Mind, 2nd edn. (Routledge, New York, 2010)

[3] Annika H. G. Peter, Dark Matter: A Brief Review (2011) arXiv:1201.3942 